\title{
Une acquisition du Centre International Blaise Pascal : le testament d'Antoinette Fontfreyde
}

\section{Régine Pouzet}

\section{(2) OpenEdition}

\section{Journals}

Édition électronique

URL : http://journals.openedition.org/ccibp/557

DOI : $10.4000 /$ ccibp. 557

ISSN : 2493-7460

\section{Éditeur}

Centre international Blaise Pascal

\section{Édition imprimée}

Date de publication : 5 avril 1997

Pagination : 6-9

ISSN : 0249-6674

\section{Référence électronique}

Régine Pouzet, « Une acquisition du Centre International Blaise Pascal : le testament d'Antoinette

Fontfreyde ", Courrier du Centre international Blaise Pascal [En ligne], 19 | 1997, mis en ligne le 06 janvier 2016, consulté le 15 septembre 2020. URL : http://journals.openedition.org/ccibp/557

Ce document a été généré automatiquement le 15 septembre 2020.

Centre international Blaise Pascal 


\title{
Une acquisition du Centre International Blaise Pascal : le testament d'Antoinette Fontfreyde
}

\author{
Régine Pouzet
}

1 Le Centre International Blaise Pascal a acquis, en 1995, une expédition du testament d'Antoinette Fontfreyde, grand-mère maternelle de Blaise Pascal. La pièce, datée du 17 juin 1627, est composée de onze pages, soit cinq feuillets, format 370 X 220, écrits rectoverso. La mention inscrite à la dernière page indique que la testatrice a, elle-même, demandé cette expédition auprès du notaire royal, Ameilhon, dépositaire de la minute ${ }^{1}$.

2 Antoinette Fontfreyde est fille d'Antoine Fontfreyde, dit Saulzet, un riche marchand de Clermont, qui a acquis, en 1568, le lieu noble de la Vieille-Varvasse sous Vialleveloux, et qui se fera qualifier de seigneur de Vialleveloux. En 1565, il est receveur des deniers de rachat des biens du clergé à Clermont. La mère d'Antoinette est Jacquette Durand, fille d'un marchand libraire de Clermont. On connaît à Antoinette Fontfreyde quinze frères et sœurs ${ }^{2}$.

3 Elle épouse Victor Begon, marchand bourgeois de Clermont, fils de Bonnet Begon, lieutenant général en la châtellenie de Gerzat, et d'Annette Vidal. De ce mariage nous lui connaissons trois enfants: Jean, Gilberte et Antoinette, qui sera la mère de Blaise Pascal.

4 Antoinette Fontfreyde fait son testament, sans être pressée par la maladie, soucieuse seulement de régler ses affaires, et cela tout en respectant, dit-elle, la volonté de son mari. Elle habite paroisse Saint-Pierre ayant quitté, à une date que nous ignorons, la paroisse Saint-Adjutor où a été baptisée sa fille Antoinette. C'est dans l'église SaintPierre que son mari, Victor, a été inhumé. C'est dans son tombeau qu'elle souhaite être ensevelie " quand il plaira à Dieu de l'appeler de ce monde ».

5 Antoinette est veuve depuis juin 1615. Victor Begon, échevin cette année-là, est mort entre deux réunions du conseil de ville, celle du 2 juin, qu'il préside, assisté de ses deux collègues échevins, et celle du 23 juin, où, dans le compte-rendu des délibérations, son nom est suivi du mot « décédé ». Il a peut-être été victime d'une de ces épidémies qui 
ravageaient périodiquement la province d'Auvergne. En effet, lors du conseil du 11 juillet 1615, les deux échevins, Gilbert de Bretanges et Antoine Savaron, demanderont que l'on prenne des mesures d'hygiène : «il serait besoin de pourvoir à la santé de cette ville attendu la maladie qu'il y a quant à présent, et le grand nombre de pauvres étrangers qui y affluent de toutes parts ${ }^{3}$ » (3E 113 DEP BB 70)

6 La testatrice a perdu sa fille aînée, Gilberte, épouse de Charles de Pierrefitte, seigneur de Bosredon et de Rochevert, morte en couches, le $1^{\text {er }}$ juin 1609. Elle venait de donner naissance à une petite fille, qui sera baptisée, huit jours après les obsèques de sa mère, dans la même église cathédrale ${ }^{4}$. L'année 1626, Antoinette perd sa deuxième fille, prénommée comme elle, Antoinette, l'épouse d'Etienne Pascal, la mère de Gilberte, de Blaise et de Jacqueline Pascal ${ }^{5}$. Il lui reste son fils Jean, conseiller et élu pour le roi en l'élection de Clermont. Il a épousé Jacquette Durant, fille d'un trésorier de France à Riom dont, en 1627, il a déjà cinq enfants, nés entre 1619 et 1624.

7 Comme il est d'usage dans tous les testaments du temps, Antoinette Fontfreyde fonde des messes pour le salut de son âme. Marque de piété, et aussi d'une aisance certaine, ce sont cinquante messes basses qui devront être dites, pour elle, dans cinq églises de la ville. Puis elle énumère les legs dont sera chargée sa succession.

8 En première place figurent les enfants d'Étienne Pascal, « au défaut de feue damoiselle Antoinette Begon, leur mère ». Au moment où Antoinette Fontfreyde rédige son testament, Gilberte, Blaise et Jacqueline ont respectivement sept ans, quatre ans et un an. Gilberte, peut-être parce qu'elle est l'aînée, ou parce qu'elle est la préférée, recevra, lorsqu'elle se mariera, 3000 livres tandis que son frère Blaise et sa sœur cadette, Jacqueline, auront chacun, pour leur part, 2000 livres. Cette façon de favoriser une fille est rare dans les testaments de l'époque, où ce sont les garçons qui, généralement, reçoivent les legs les plus importants. On note que la testatrice ne prévoit rien pour Antoinette de Pierrefite, sa première petite-fille et filleule. Peut-être l'enfant est-elle décédée entre temps. Les autres legs, beaucoup plus modestes, concernent une de ses nièces, Antoinette Esparvier, fille de sa sœur Anne, décédée ; mais aussi les servantes de Gilberte et de cette nièce. Gilberte, qui n'a que sept ans, a donc une servante attachée à son service. Enfin, 100 livres doivent contribuer à faire réparer l'église Saint-Pierre, dont on sait qu'elle était fort délabrée. Quant à Jean Begon, son fils, elle l'institut son héritier universel, à charge pour lui de payer legs et fondations de messes. Moyennant quoi il héritera, comme l'a souhaité son père Victor Begon, de tous les biens qu'elle délaissera par son décès.

Elle prend soin de désigner un exécuteur testamentaire, auquel elle donne plein pouvoir de prendre, sur les biens qu'elle laissera, meubles ou immeubles, ce qu'il faudra pour exécuter les legs, dans le cas où son héritier universel négligerait de le faire. Doutait-elle de l'honnêteté de son fils? Craignait-elle seulement qu'il fût négligent ? De fait, elle pressentait bien l'avenir, mais choisissait mal son exécuteur testamentaire puisqu'il n'usera pas de ses pouvoirs. Jacques Chardon, avocat au présidial, son neveu par alliance, qu'elle a choisi pour cette tâche, meurt à Clermont, paroisse Saint-Genès, le 30 mars 1644, sans avoir fait exécuter les legs concernant les enfants d'Étienne. Dans le contrat de mariage de Gilberte en 1641, figurent les 3000 livres que doit, à la future épouse, l'héritier d'Antoinette Fontfreyde. Nous ne savons si elles ont été payées. On peut en douter, car, soixante-dix-huit ans plus tard, les legs aux enfants Pascal, qui, en vertu des termes du testament, se sont cumulés sur la survivante, Gilberte, restent encore dus par les Begon aux héritiers de celle-ci. En effet le testament d'Antoinette 
Fontfreyde sera cité dans un acte du 28 décembre $1705^{6}$, où nous apprenons que la dette des Begon à l'égard des héritiers des Pascal dérive, "en partie ${ }^{7}$ ", du "testament d'Antoinette Fontfreyde, veuve de Victor Begon, du $17^{\mathrm{e}}$ juin 1627 ».

Une autre clause de son testament ne sera pas respectée. Elle souhaitait être inhumée dans l'église Saint-Pierre, au tombeau où avait été enseveli son mari. Or c'est dans l'église de Gerzat qu'elle sera ensevelie ${ }^{8}$. Deux lignes lui sont consacrées dans un "Extrait des registres des sépultures de l'église paroissiale de Gerzat », "Honnête femme dame Antoinette Fontfreyde est décédée le 9 janvier 1632 ». Ainsi on n'aurait pas tenu compte de ce qui constitue, dans tous les testaments de l'époque, un élément important des dernières volontés du testateur? Faut-il en déduire l'indifférence de Jean Begon à l'égard des vœux de sa mère? Peut-être pas. Des circonstances particulières et les malheurs du temps peuvent expliquer cette inhumation à Gerzat. Clermont et ses environs ont été accablés par la peste pendant l'année 1631. Les familles aisées ont quitté la ville et se sont réfugiées dans leurs domaines, à la campagne, ou dans les villes voisines. Antoinette Fontfreyde se serait réfugiée avec ses enfants et ses petits-enfants Begon, dans ce village de Gerzat, proche de Clermont, où elle possède un domaine. Elle y est encore en ce début d'année 1632, où elle va mourir. Depuis trois mois la maladie a sensiblement régressé à Clermont. Mais elle s'est répandue aux alentours et les responsables de la ville exigent une quarantaine avant de laisser entrer dans leurs murs les habitants qui se sont retirés dans des lieux maintenant infectés. Gerzat est peut-être de ceux-là, on cite Saint-Julien de Coppel, Volvic, Blanzat, Boisséjour, Beaumont, Chadrat et Cournon'. Dans ce cas, il aura pu être impossible de transporter le corps d'Antoinette Fontfreyde dans l'église Saint-Pierre de Clermont.

11 Elle n'aura pas eu, sans doute, la consolation d'avoir auprès d'elle les enfants de sa fille Antoinette. Étienne Pascal, élu au conseil de ville de Clermont depuis le 3 janvier 1631, a dû abandonner ses fonctions aux assemblées de la ville dès la mi-mai ${ }^{10}$, le roi ayant imposé aux magistrats chargés de la justice de se transporter dans les villes voisines épargnées par la maladie. Le Présidial est à Vic-le-Comte ${ }^{11}$, la Cour des aides à Pont-duChâteau ${ }^{12}$. Les enfants d'Étienne ont-ils suivi leur père dans cette dernière ville ? Sontils, le mal s'étant éloigné, retournés à Clermont, où la maladie aurait enlevé cinq à six mille personnes ${ }^{13}$ ? Étienne que l'état sanitaire de la ville pouvait inquiéter a peut-être emmené directement ses enfants à Paris. Selon Gilberte, c'était en novembre $1631^{14}$. Elle ne reverra pas sa grand-mère qui semblait lui vouer une affection particulière.

\section{Texte du testament d'Antoinette Fontfreyde}

12 Notre transcription du testament d'Antoinette Fontfreyde respecte strictement la graphie du document. Le lecteur ne devra pas s'étonner de l'absence d'accent aigu sur l'e final, prononcé é, l'emploi de c pour s. Nous restituons entre crochets, les syllabes abrégées. Nous avons modernisé la ponctuation.

L'an mil six cents vingt sept et le dix septiesme jour du mois de jung par devant le notaire royal soubz[sig]ne et tesmoingtz bas nommes, c'est comparue et pr[esen]tee en personne honneste femme Anthoinette Fontfraide, vefve de honnorable homme Victour Begon, quant vivoict son mary, dame de ses droictz et n'estant en la puissance d'aucun ainsin qu'elle a dict, ha[bita]nte de ceste ville de Clermont, laquelle de son bon gre etc. sans aucune persuasion ne contraincte, sayne de corps et d'esprit, et ce souvenant de la mort et de l'incertitude de l'heure d'icelle et ne 
voulant decedder de ce monde passager sans avoir dispose de ses biens et de ceulx que / lui ont este delaisses par sondict mary et dezirant d'accomplir sa voullonte. A ces causes et autres a ce la mouvant, a faict et ordonne son testament et ordonnance de derniere vollonte ainsi que s'ensuict. Premierement a faict le signe de la Saincte+Crois sur sa personne en dizant au nom du pere et du filz et du sainct esprit et c'estant recommendee a dieu tout puissant par l'intercession de son tres cher filz et de la glorieuze Vierge Marie et de tous les saincts et sainctes de paradis, a donne son ame a dieu et son corps a la terre, quant luy plairrat de l'appeller de ce monde; vollu qu'il soict sepulture en l'esglize parrochialle de Sainct Pierre / au tumbeau ou sond. mary fust ensepveli, et pour la forme de sa sepulture et honneurs funebres s'en remet entierement a la vollonte et discreition de son heritier cy appres nomme, sy n'est qu'elle veult et entend que pour le sallut et remede de son ame, et le jour que dieu l'aura appellee de ce monde ou le landemain de sondict decedz, il soict dict et celebre cinquante messes basses, savoir dix a lad. esglize Sainct Pierre dud. Clermont, dix a l'esglize des Cordeliers, dix a l'esglize des Carmes, dix a l'esglize des Capusins, et les autres dix a l'esglize cathedrale dudict Clermont. Et / pour ces biens a donne et legue a damoizelle Gilberte Pascal, fille a noble Estienne Pascal, presidant en la cour des aides a Montferrant, la somme de trois mil livres $\mathrm{t}$ [ournoi]z, paiables lorsqu'elle ce mariera, a Blaize Pascal, son frere, la somme de deux mil livres $\mathrm{t}$ [ournoi]z, paiables lorsqu'il aura attainct l'aige de quinze ans, a Jacquette Pascal, leur sœur, la somme de deux mil livres $t$ [ournoi]z paiables aussi lorsqu'elle trouvera son party en mariaige, tous enffens dudict Pascal et de feue damoizelle Anthoinette Begon, vivant filhe de lad. Fontfraide. Veult et entend lad. testatrice que si l'un ou l'au[tr]e desd. enffens venoict a deceder que les aultres heriteront / de luy, et venant tous a decedder sans enffens ny decendans d'eulx, que lesd. choses donnees retourneront a son heritier cy appres nomme. Plus a donne ladicte Fontfraide a dame Anthoinette Esparvier, sa niepce, et femme a honnorable homme Anthoine Augier, bourghoix en ceste ville, la somme de trois cents livres paiables a lad. Esparvier deux ans appres sondict decedz. Aussy a donne comme dessus a Antoinette Auchier de Lempde, sa filheulle, la somme de trente livres paiables lorsqu'elle ce mariera. Pareille somme de trente livres a donne et legue comme dessus / a Martine Faure servante a lad. damoizelle Gilberte Pascal, paiab[le] aussy quant elle ce mariera. Semblable somme de trente livres a aussi donne comme dessus a Michelle [en blanc] servante a lad. Esparvier et aud. Augier, aussy paiable lorsqu'elle trouvera son party en mariaige. De plus a donne et legue comme dessus a ladicte esglize de Sainct Pierre de cested. ville la somme de cent livres, qu'elle veult et ordonne estre paies au chapp[it]re de ladicte esglize un an appres sond. decedz pour estre emploies aux repara[ti]ons necessaires de ladicte esglize et non ailhieurs par ledict chapp[it]re. Touttes lesquelles sommes sus donnees et leguees par lad. dame / Fontfraide, elle veult et ordonne estre paiee[s] a ch[ac]un des susnommes aux termes susd. par sondict heritier, lequel elle charge de faire les paiements desd. sommes comme aussy pour les cinquante messes a ceulx qui les diront et celebreront. Et par ce que le chef $\mathrm{e}[\mathrm{t}]$ fondement de tout bon et valable testament est l'institu[ti]on d'heritier ou heritiere, et recognoissant que sondict feu mary l'a chargee de delaisser a noble Jehan Begon, cons[eill]er et esleu pour le Roy en l'eslection de ceste ville leur filz tous les biens qu'elle delaisserat par son decedz appres les legatz cy dessus faictz aux enffens dud. Pascal au deffauld / de lad. feue damoizelle Anthoinette Begon leur mere. Elle a nomme et institue pour son heritier universel en tous et ch[ac]uns ses biens ledict noble Jehan Begon, son fils, a la charge qu'il paiera les legatz et charges cy dessus ; et pour executer et faire entretenir le $\mathrm{p}$ [rese]nt testament elle a nomme Monsieur Me Jacques Chardon, advocat en la sen[echauss]ee et siege presidial d'Auvergne en ceste ville, lequel elle a prie volloir acepter lad. charge et que au deffauld de paier et entretenir le contenu au $\mathrm{p}$ [rese]nt testament par sond. heritier, a donne plain pouvoir et puissance aud. s[ieu]r Chardon de prendre de tous et ch[ac]uns ses biens qu'elle delaissera par sond. decedz soict meubles / ou immeubles pour faire les paiementz 
et entretenir ce que dessus dezirant que le p[rese]nt testament sorte son plain et entier effect nonobstant touttes coustumes, ordonnances et formes de justice que ad ce pourroit estre contraires. Et a revoque, casse et anulle par le presant tous $\mathrm{au}[\mathrm{tr}] \mathrm{es}$ testamentz, codicilles et donna[ti]ons a cause de mort qu'elle pourroit avoir faictz par cy devant, et au paiement et entretenement de tout ce que dessus, a lad. testatrice, oblige tous et $\mathrm{ch}[\mathrm{ac}] \mathrm{uns}$ ses biens meubles immeubles $\mathrm{p}$ [rese]ntz et advenir et iceulx soubzmis etc. renonce etc. Faict a Clermont maison de lad. dame testatrice, lesd. jour / mois et an que dessus apres midy es presances de Mre Francois Alegre de La Tour, Gervais Ameilhon, Estienne Condat, Pierre de La Roche, tailheur d'habitz, Anthoine Tailhefert courdounier aud. Clermont, et Me Anthoine Bourrasset praticien, ha[bita]nt d'Auzon qui ont signe aveq lad. dame Fontfraide testatrice, a l'original des $\mathrm{p}$ [rese]ntes et le no[tai]re royal soubz[sig]ne et Helie Roche dud. Clermont qui a dict ne scavoir signer.

Expedie a ladicte dame de Fontfraide testatrice le requerant.

Ameilhon notaire royal ainsi est a l'original.

Contrôlé le XVIIIe jung 1627

[Au verso du dernier feuillet]

Testament

de dame Anthoinette de Fontfraide vefve de feu le sieur Victor Begon son espoux lad. dame testatrice.

du 17 iung 1627

\section{NOTES}

1. Cette minute n'a pu être retrouvée dans les archives du notaire Ameilhon, aux Archives départementales du Puy-de-Dôme.

2. A. de Remacle, Dictionnaire généalogique, Familles d'Auvergne, Clermont-Ferrand, 1995, II.138-139

3. Arch. Départ. Puy-de-Dôme, 3E 113 DEP BB 70.

4. Arch. départ. Puy-de-Dôme, 3 E 113 DEP fds I.1.

5. Les registres de sépulture de la paroisse Saint-Pierre n'ayant pas été conservés pour les années antérieures à 1649, nous n'avons, pour préciser cette date, que le témoignage de Gilberte Pascal (Pascal, Cuvres, éd. Jean Mesnard, Desclée de Brouwer, 1964, I.570). Ce décès n'a pu survenir qu'après le 13 février 1626, car, à cette date, nous trouvons la signature de la mère de Pascal au bas d'une mention de baptême du registre paroissial de l'église Saint-Genès (Arch. dép. Puy-deDôme, 6 E 113/6).

6. Notaire Lascrotas, Arch. dép. du Puy-de-Dôme, 5 E 19 DEP 443.

7. D'autres dettes se sont ajoutées à celle-ci. Voir l'allusion de Gilberte Pascal, à un trésorier de France, dont la famille doit beaucoup aux Périer (Pascal, Euvres, éd. Jean Mesnard, I. 1083). Ce personnage, auquel Florin Périer se proposait de prêter encore de l'argent, est, vraisemblablement, un des fils de Jean Begon, Annet.

8. Arch. dép. du Puy-de-Dôme, Reg. cath. 6 E 164/1

9. ibid., 3E 113 DEP.I. BB 79

10. ibid.

11. Manry, Sève, Chaulanges, L'histoire vue de l'Auvergne, Clermont-Ferrand, 1959.

12. Peghoux, Sur les épidémies qui ont ravagé l'Auvergne, Clermont-Ferrand, 1835, p. 456.

13. ibid.

14. Pascal, Cuvres, éd. Jean Mesnard, I. 659. 
INDEX

Mots-clés : Fonfreyde (Antoinette), testament

Keywords : Fonfreyde (Antoinette), will

\section{AUTEUR}

RÉGINE POUZET

Université Blaise Pascal 